\title{
A Rare Case of Squamous Cell Carcinoma Arising in Mature Cystic Teratoma
}

\author{
Andi Kurniadi ${ }^{1}$ D, Ali Budi Harsono ${ }^{1}$, Kemala Mantilidewi ${ }^{1 *}$ (D), Muhamad Khalif Anfasa ${ }^{1}$, Maya Noor Fitriana ${ }^{2}$, \\ Bethy Hernowo ${ }^{2}$ i \\ ${ }^{1}$ Department of Obstetrics and Gynecology, Faculty of Medicine, Universitas Padjadjaran, Dr. Hasan Sadikin General Hospital, \\ Bandung, Indonesia; ${ }^{2}$ Department of Anatomical Pathology, Faculty of Medicine, Universitas Padjadjaran-Dr. Hasan Sadikin \\ General Hospital, Bandung, Indonesia
}

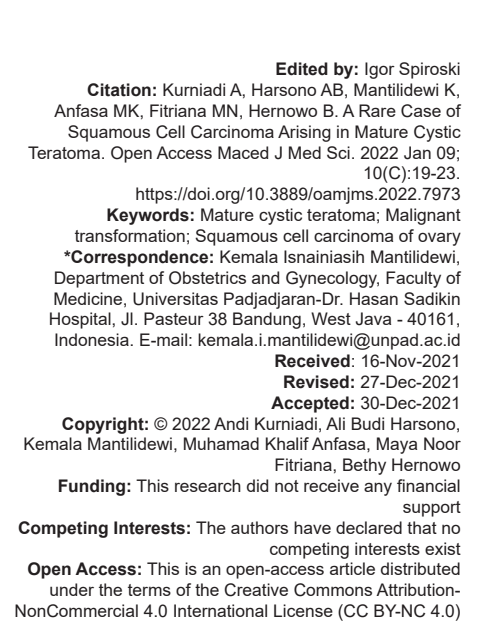

\section{Abstract}

BACKGROUND: Mature cystic teratoma (MCT) is a common benign adnexal tumor contributing to about $20 \%$ of al ovarian tumors, and it may occur in $10-20 \%$ of women during their lifetime. Although the nature of MCT of the ovary is benign, about $1-2 \%$ of MCT undergo malignant transformation.

CASE REPORT: Here, we report a rare case of squamous cell carcinoma (SCC) arising in MCT. This one rare case report of SCC arising in MCT showed satisfactory response to adjuvant chemotherapy. Careful routine evaluation is needed for possible recurrency.

CONCLUSION: This patient was found with infiltration to the urinary bladder and concluded as stage IIB; therefore the surgeon decided to give adjuvant chemotherapy with paclitaxel/carboplatin. After given three cycles of paclitaxe carboplatin chemotherapy the patient showed favorable outcome, as the CT scan evaluation showed no residual mass.

\section{Introduction}

Teratoma is a germ cell tumor that may be composed of either mature or immature tissues arising from the three germ cell layers, namely ectoderm, mesoderm, or endoderm [1]. About $95 \%$ of all ovarian teratomas are mature cystic teratoma (MCT). MCT is a common benign adnexal tumor contributing to about $20 \%$ of all ovarian tumors, and it may occur in $10-20 \%$ of women during their lifetime [2], [3].

Although the nature of MCT of the ovary is benign, about $1-2 \%$ of $\mathrm{MCT}$ undergo malignant transformation [4]. Malignant transformation can occur in various histological types, namely: squamous cell carcinoma (SCC), adenocarcinoma, small cell carcinoma, sarcoma, malignant melanoma, and mixed histology. Among all of them, the most common SCC transformation in MCT is the most common, accounting for about $75-90 \%$ of all malignant transformation, followed by adenocarcinoma $(7 \%)$ and sarcoma (7\%) [2], [3]. SCC transformation in MCT is predicted to be a continuous process of squamous metaplasia, atypical hyperplasia, carcinoma in situ, interstitial infiltration and invasive carcinoma [2]. The tumor may arise from any of three germ cell layers present in the teratoma, and has been observed adjacent to both normal and metaplastic cells [4]. Here, we report a rare case of SCC arising in MCT.

\section{Case Presentation}

This is a case of a 70-year-old female in Bandung City, West Java, Indonesia, diagnosed with suspected malignant ovarian tumor. Based on history taking, the patient felt difficulty in urinating and abdominal bloating for one month. Other complaints such as decrease appetite, weight loss, abdominal enlargement, and defecation problem were denied by the patient. She initially had been treated by antibiotics for her urinary problem by an internist. Since there was 
no improvement after antibiotics treatment, the internist suggested abdominal ultrasonography examination. The result showed a mass suggested a teratoma (Figure 1). The patient then was consulted to an obstetrician and the obstetrician concluded the patient has a high possibility of a malignancy of the ovary. Therefore, the patient was referred to an oncology gynecologist.

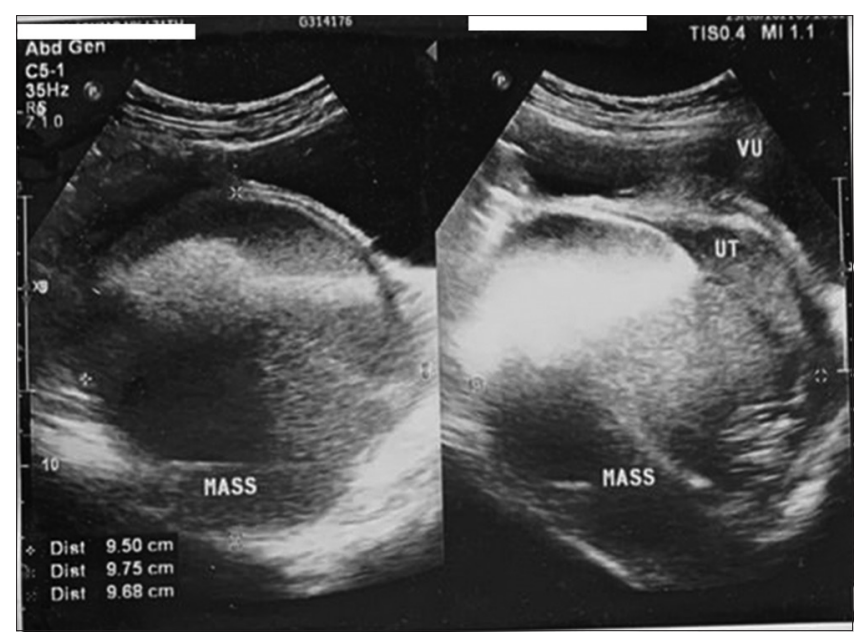

Figure 1: Lower abdomen ultrasonography showed pelvic mass suspected ovarian cyst. UT: uterus; VU: Urinary bladder

From further asssessment by the oncology gynecologist, the patient underwent examination by contrast-enhanced CT Scan of abdomen and pelvis, and the result showed suspected a right sided ovarian mass suggestive a teratoma (Figure 2a), with possible infiltration to bladder (Figure 2b). All examination of liver, gall bladder, spleen, bilateral kidneys, and bladder showed no abnormalities. There were no enlargement of the paraaorta and bilateral parailiaca lymph nodes.

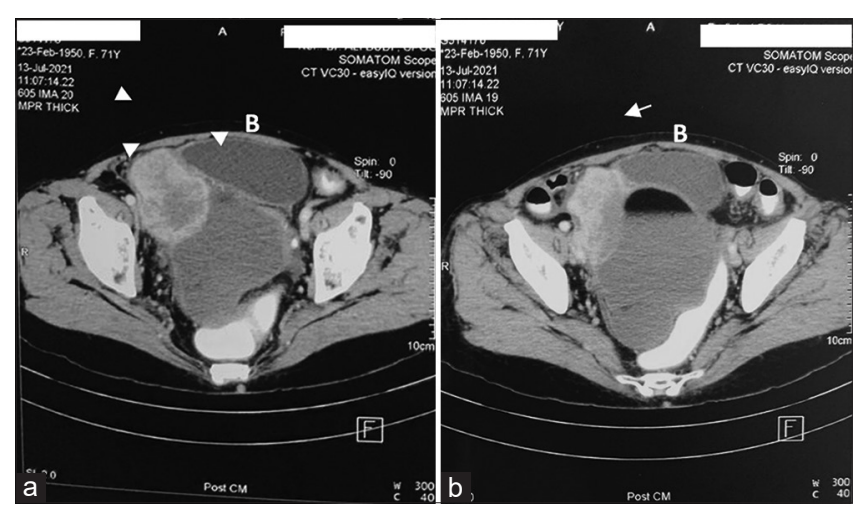

Figure 2: Contrast-enhanced Computed tomography Scan of whole abdomen and pelvis. (a) A right sided ovarian mass (arrow head). (b) Possible infiltration to bladder showed by partial irregular mucosal bladder (arrow). B: Bladder

Complete laboratory examination of routine hematology, ureum, creatinin, and fasting blood glucose were all within normal limit. Serum tumor marker of carcinoembryonic antigen (CEA) was slightly high (7.6 ng/mL), and CA 125 was $36 \mathrm{U} / \mathrm{mL}$.
During operation, the surgeon found the mass was originated from the right ovary, size $12 \times 10 \mathrm{~cm}$ and was highly adhered to bladder. Appendix was enlarged and adhered to the mass. Mass was successfully resected by performing right salpingo-oophorectomy. The surgeon also performed appendectomy and did frozen section.

The frozen section result based on macroscopic findings were a right ovary size $12.5 \times 10 \times 9 \mathrm{~cm}$, containing porridge-like fluid, mixed with solid part and hair-like structures. The right fallopian tube was found with no abnormalities. Microscopically on the ovary showed a cystic structure lined by a stratified squamous epithelium, and also hair follicles. Tumor cells arrange in the form of solid sheets, trabecular, and nests in desmoplastic stroma with large area of necrosis. The nuclear feature shows polymorphic, hyperchromatic, and prominent mitosis. The feature of paraffin block of frozen section specimen is shown on Figure 3a-d. Some malignant indicators were found, such as tumor cells that had invaded the lymph vessels, and the tumor cells were also infiltrated the fallopian tube, as well as the appendix. Final conclusion of the frozen section was MCT with neuroendocrine carcinoma of the right ovary, right fallopian tube and appendix.

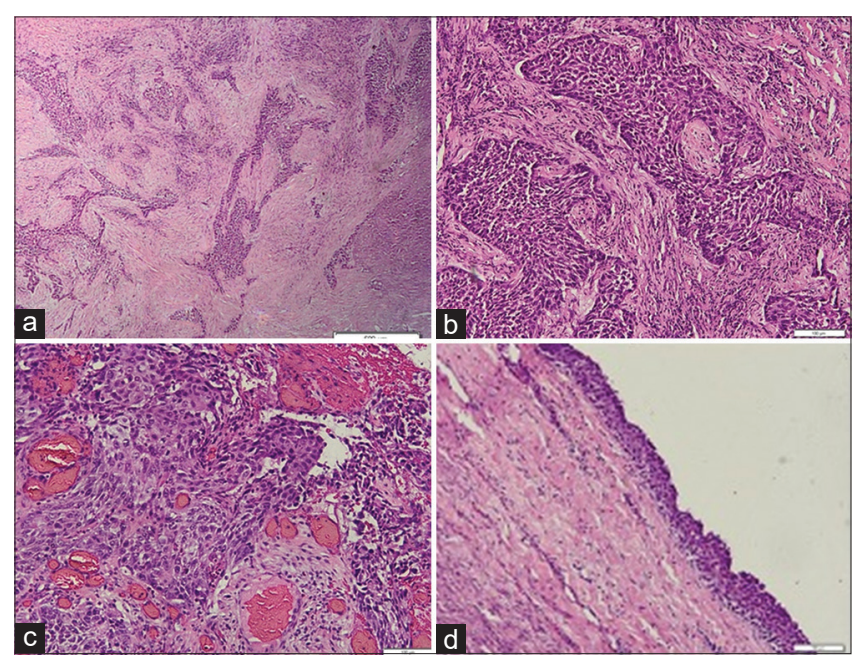

Figure 3: (a) The tumor mass in the ovary was arranged in nest and trabeculae form in low-power view. (b and c) High-power view of tumor nest. (d) The cyst wall was lined by stratified squamous epithelial. Scale bars, $100 \mu \mathrm{m}$

With these frozen section findings the patient then underwent surgical staging, and the surgeon performed hysterectomy and left salpingooophorectomy, lymphadenectomy of paraaorta and bilateral pelvic lymph nodes, and omentectomy. During exploration, the surgeon found mass has infiltrated the bladder and decided to perform partial resection and repair of the urinary bladder.

All specimen then underwent histopathological examination. The right ovary, the right fallopian tube, and the appendix showed same result as the frozen section. The cervix, uterus, left fallopian tube, omentum, 
paraaorta lymph node, and the bilateral pelvic lymph nodes showed no abnormalities. There is an invasion of tumor cells in bladder (Figure 4). Found the left ovary with follicular cyst. Final conclusion showed MCT of the right ovary with carcinoma of neuroendocrine on the right ovary, right fallopian tube, and appendix.

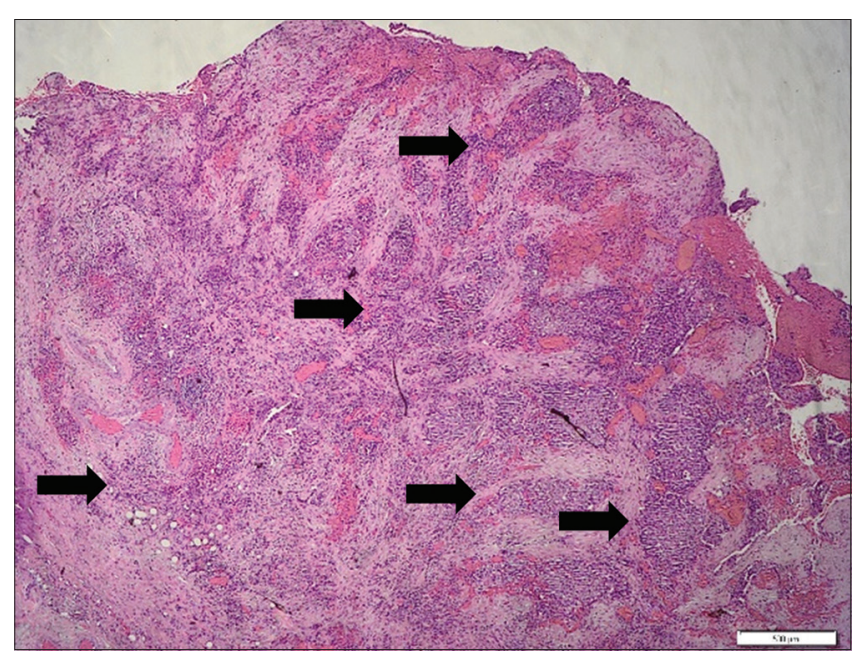

Figure 4: Tumor nest has been infiltrated to bladder (shown by arrow). Scale bar, $500 \mu \mathrm{m}$

The pathologist suggested further examination by immunohistochemistry $(\mathrm{IHC})$. The $\mathrm{IHC}$ result was shown in Figure 5. The IHC of cytokeratin showed positive result (Figure 5a), Ki67 showed positive high proliferation (Figure 5b), and negative in Synapthophysin and Chromogramin staining (Figure 5c and $\mathrm{d}$, respectively). The IHC concluded the tumor is carcinoma with final result implicated an SCC arising in MCT.

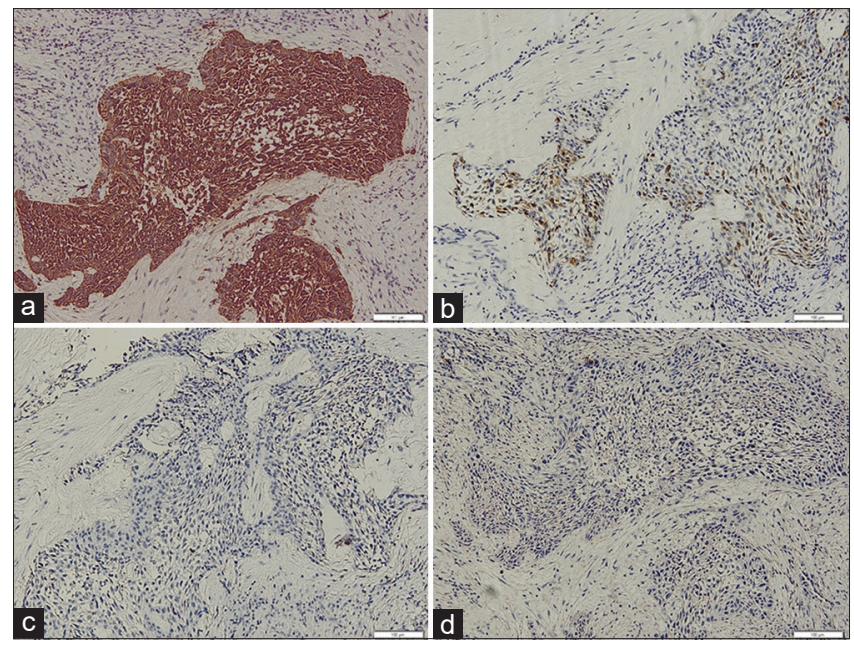

Figure 5: (a) Positive expression of cytokeratin. (b) Positive expression of Ki67. (c) Negative expression in Synapthophysin. (d) Negative expression in Chromogramin staining. Scale bars, $100 \mu \mathrm{m}$

The patient then received adjuvant chemotherapy post surgey with three cycles of paclitaxel carboplatin chemotherapy, with favorable outcome. Computed tomography (CT) Scan evaluation for possible metastasis appeared no residual mass after the adjuvant chemotherapy. The patient was planned for additional three cycles of adjuvant chemotherapy and routine evaluation for possible recurrences.

\section{Discussion}

Ovarian germ cell tumors are a histologically heterogeneous group of tumors that arise from the same origin, namely primordial germ cells of the embryonic gonad.[5] In detail, germ cell tumors of the ovary can be classified into three categories: primitive germ cell tumors, biphasic or triphasic teratoma, monodermal teratoma, and somatic-type tumors associated with dermoid cysts.[6,7] Ovarian germ cell tumors account for $20-25 \%$ of all ovarian neoplasms.[8] Teratoma may be composed of either mature or immature tissues.[1] About $95 \%$ of all ovarian teratomas are mature cystic teratoma (MCT), a common benign adnexal tumor contributing to about $20 \%$ of all ovarian tumors. MCT may occur in $10-20 \%$ of women during their lifetime[2,3], and may occur at any age, but the peak incidence is in reproductive age[9].

Although the nature of MCT of the ovary is benign, about $1-2 \%$ of MCT undergo malignant transformation [4]. Malignant transformation can occur in various histological types; namely SCC, adenocarcinoma, small cell carcinoma, sarcoma, malignant melanoma, and mixed histology. Among all of them, SCC transformation in MCT is the most common, accounting for about $75-90 \%$ of all malignant transformation, followed by adenocarcinoma (7\%), and sarcoma (7\%) [2], [3]. In this present case, we elaborate our experience in a case of SCC transformation in MCT.

SCC transformation in MCT is predicted to be a continuous process of squamous metaplasia, atypical hyperplasia, carcinoma in situ, interstitial infiltration , and invasive carcinoma [2]. The tumor may arise from any of three germ cell layers present in the teratoma and has been observed adjacent to both normal and metaplastic cells [4].

There are several hypotheses about the cause of this malignant transformation. Prolonged exposure of the pelvic cavity to carcinogens and high-risk HPV infections was associated with SCC transformation in MCT. Several studies have shown that p53 and p16 mutations may be involved in this malignant transformation [1], [2], [10] Cyclooxygenase-2 [COX-2] expression has also been detected in SCC transformation of MCT, but not in benign MCT and adenocarcinoma transformation of MCT. Therefore COX-2 is only involved in the malignant transformation of MCT into SCC [11].

Pre-operative detection of MCT malignant transformation is difficult due to the lack of specific signs 
and symptoms, tumor markers, and imaging findings. In the early stages, the patients are usually asymptomatic and tumor is often found incidentally during a physical examination or postoperative pathological examination. Symptoms such as palpable abdominal or pelvic mass, abdominal pain, and bloating are often found in more advanced tumor stages [1], [2], [3], [12] Cachexia, weight loss, and other constitutional symptoms may also occur in chronic advanced cases [12]. Occasionally, the presenting complaints might be gastrointestinal symptoms such as constipation, diarrhea, or rectal bleeding, and urinary symptoms such as frequency that occur due to invasion of nearby organs [3]. Acute abdomen may also be present due to tumor torsion or rupture, and this incident may calls for an emergency surgery [1], [2]. Our patient was presented with urinary symptoms of difficulty of urination, which is evident by intra-operative findings of mass infiltrating the urinary bladder. Nonspecific symptoms of bloating also felt by this patient. Adherence of a part of small intestine to the mass may lead to acute intestinal obstruction [12]. The size of a malignant ovarian MCT is usually larger than that of a benign MCT, and patients with malignant tumors are usually older than those with benign MCT [13].

As MCT is a fairly common ovarian neoplasm, thus promoting emphasis on pre-operative malignancy risk assessment of these tumors. Predictive factors of MCT malignant transformation include old age ( $>45$ years old), large tumor size, levels of several serum tumor markers (SCC antigen, CA125, CA19-9, and CEA), and larger solid components in MCT [1], [3], [12], [14].

Compared to CA125 and CA19-9, serum CEA and SCC antigen are better screening markers. Out of the two, SCC appears to be a more relevant marker for preoperative risk assessment and for helping to differentiate between a teratoma and SCC. However, serum SCC alone could not exclude malignant transformation. In this patient, the tumor marker evaluated was CEA and CA 125 , thereby examinations of other tumor markers may be considered in patients with highly predictive for malignancies. Radiological features that indicate malignant transformation are solid component that extends transmurally invading adjacent structure, irregular invasion through the septa or peritoneal area, necrosis, and hemorrhage [1], [14]. Moreover, vascularization in Doppler imaging should lead to malignancy suspicion [4], [15].

Although not considered as gold standard for MCT cases, frozen section should be warranted in the presence of malignant predictive factors. If suspicions of malignancy were found on frozen section, the surgeon might lean toward performing staging laparotomy in place of conservative surgery. Therefore, executing a frozen section may permit patients to avoid second surgery [1], [3]. In our case, the surgeon had already suspected malignancy and performed frozen section that revealed the highly suspicious MCT with malignancy characteristics.
Laparoscopic surgery is a novel and preferred technique for gynecological surgery, whenever possible. Successful laparoscopic removal of MCTs without spillage is already challenging, therefore an open approach with exploratory laparotomy is preferred whenever a strong suspicion of malignancy arises to prevent inadvertent tumor upstaging by intraperitoneal rupture [1], [4]. Complete surgical excision and proper staging are crucial to the patient's prognosis and treatment plan and, therefore, should be performed at the initial surgery or as soon as possible.[4,12] Surgical cytoreduction has been associated with survival improvement and should also be considered.[4] Fertility sparing surgery with unilateral salpingo-oophorectomy is safe and justifiable in SCC transformation of MCT if the patient is younger than 45 years old with stage IA/IC disease. For postmenopausal women, the treatment should be hysterectomy with bilateral salpingo-oophorectomy with complete surgical staging [2], [12].

To date, there is still no available consensus concerning adjuvant treatment modalities such as chemotherapy or radiotherapy and their effectiveness on SCC transformation of MCTs [1], [12]. Initial chemotherapy regimen recommended for ovarian germ cell tumor and epithelial ovarian cancer is bleomycin/ etoposide/cisplatin and paclitaxel/carboplatin (TC), respectively [2]. Considering adjuvant chemotherapy for SCC transformation in MCT, most studies encourage the use of platinum-based chemotherapy because of its effectiveness as adjuvant therapy in epithelial ovarian and cervical SCC.

Adjuvant chemotherapy is not beneficial in stage I or II diseases, but it may improve prognosis of patients with stage III and IV diseases [1], [2], [14] Prognosis of this tumor depends mainly on its stage. Other factors affecting prognosis are tumor grade, growth pattern, capsular rupture, and vascular invasion [4]. This patient was found with infiltration to the urinary bladder and concluded as stage IIB; therefore, the surgeon decided to give adjuvant chemotherapy with paclitaxel/ carboplatin. After given three cycles of paclitaxel carboplatin chemotherapy the patient showed favorable outcome, as the CT scan evaluation showed no residual mass. The patient was planned to receive additional three cycles of paclitaxel carboplatin chemotherapy and advised for routine follow-up consisting of physical examination, ultrasonography, and CT-scan examination to evaluate any possible recurrences.

\section{Authors' Contributions}

All authors contributed equally. 


\section{References}

1. Chiang AJ, Chen MY, Weng CS, Lin H, Lu CH, Wang PH, et al. Malignant transformation of ovarian mature cystic teratoma into squamous cell carcinoma: A taiwanese gynecologic oncology group (TGOG) study. J Gynecol Oncol. 2017;28(5):e69. https:// doi.org/10.3802/jgo.2017.28.e69

PMid:28657230

2. Li C, Zhang Q, Zhang S, Dong R, Sun C, Qiu C, et al. Squamous cell carcinoma transformation in mature cystic teratoma of the ovary: A systematic review. BMC Cancer. 2019;19(1):217. https://doi.org/10.1186/s12885-019-5393-y

PMid:30866852

3. Srivastava P, Dawson L, Mandal AK. Squamous cell carcinoma arising in mature cystic teratoma with sigmoid invasion. J Cancer Res Ther. 2015;11(4):1024. https://doi. org/10.4103/0973-1482.148692

PMid:26881587

4. Dos Santos L, Mok E, lasonos A, Park K, Soslow RA, Aghajanian $\mathrm{C}$, et al. Squamous cell carcinoma arising in mature cystic teratoma of the ovary: A case series and review of the literature. Gynecol Oncol. 2007;105(2):321-4. https://doi. org/10.1016/j.ygyno.2006.12.008

PMid:17240432

5. Shaaban AM, Rezvani M, Elsayes KM, Baskin H, Mourad A, Foster BR, et al. Ovarian malignant germ cell tumors: Cellular classification and clinical and imaging features. Radiographics. 2014;34(3):777-801. https://doi.org/10.1148/rg.343130067 PMid:24819795

6. Pectasides D, Pectasides E, Kassanos D. Germ cell tumors of the ovary. Cancer Treat Rev. 2008;34(5):427-41. https://doi. org/10.1016/j.ctrv.2008.02.002

PMid:18378402

7. Low JJ, Ilancheran $\mathrm{A}, \mathrm{Ng}$ JS. Malignant ovarian germ-cell tumours. Best Pract Res Clin Obstet Gynaecol. 2012;26(3):34755. https://doi.org/10.1016/j.bpobgyn.2012.01.002 PMid:22301054

8. Smith $\mathrm{HO}$, Berwick M, Verschraegen CF, Wiggins $\mathrm{C}$, Lansing L,
Muller CY, et al. Incidence and survival rates for female malignant germ cell tumors. Obstet Gynecol. 2006;107(5):1075-85. https:// doi.org/10.1097/01.AOG.0000216004.22588.ce

PMid: 16648414

9. Kim MJ, Kim NY, Lee DY, Yoon BK, Choi D. Clinical characteristics of ovarian teratoma: Age-focused retrospective analysis of 580 cases. Am J Obstet Gynecol. 2011;205(1):32. e1-4. https://doi.org/10.1016/j.ajog.2011.02.044

PMid:21507373

10. Chiang A, Chen D, Cheng J, Chang T. Detection of human papillomavirus in squamous cell carcinoma arising from dermoid cysts. Taiwan J Obstet Gynecol. 2015;54(5):559-66. https://doi. org/10.1016/j.tjog.2015.08.008

PMid:26522111

11. Gadducci A, Guerrieri ME, Cosio S. Squamous cell carcinoma arising from mature cystic teratoma of the ovary: A challenging question for gynecologic oncologists. Crit Rev Oncol Hematol. 2019;133:92-8. https://doi.org/10.1016/j.critrevonc.2018.10.005 PMid:30661663

12. Maharjan S. Mature cystic teratoma of ovary with squamous cell carcinoma arising from it. Clin Case Rep. 2019;7(4):668-71. https://doi.org/10.1002/ccr3.2062

PMid:30997060

13. Desouki M, Fadare $O$, Chamberlain $B$, Shakir $N$, Kanbour-Shakir A. Malignancy associated with ovarian teratomas: Frequency, histotypes, and diagnostic accuracy of intraoperative consultation. Ann Diagn Pathol. 2015;19(3):1036. https://doi.org/10.1016/j.anndiagpath.2015.02.004 PMid:25773307

14. Indulkar ST, Khare MS, Shah VB, Khade AL. Squamous cell carcinoma arising in mature teratoma of the ovary masquerading as abdominal tuberculosis. J Midlife Health. 2018;9(1):44-6. https://doi.org/10.4103/jmh.JMH_81_17 PMid:29628730

15. Avcı S, Selcukbiricik F, Bilici A, Özkan G, Özağarı AA, Borlu F. Squamous cell carcinoma arising in a mature cystic teratoma. Case Rep Obstet Gynecol. 2012;2012:314535. https://doi. org/10.1155/2012/314535

PMid:23320213 